This article was downloaded by: [The University of Manchester]

On: 20 July 2010

Access details: Access Details: [subscription number 915545543]

Publisher Psychology Press

Informa Ltd Registered in England and Wales Registered Number: 1072954 Registered office: Mortimer House, 3741 Mortimer Street, London W1T 3JH, UK

\section{APHASIOLOGY}

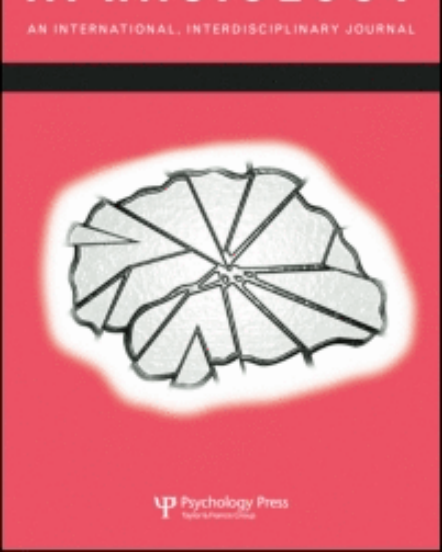

\section{Aphasiology}

Publication details, including instructions for authors and subscription information:

http://www.informaworld.com/smpp/title content=t713393920

\section{Implementing and evaluating aphasia therapy targeted at couples' conversations: A single case study}

Ray Wilkinson ${ }^{\mathrm{a}}$; Karen Bryan ${ }^{\mathrm{b}}$; Sarah Lock ${ }^{\mathrm{c}}$; Karen Sage ${ }^{\mathrm{a}}$

${ }^{\mathrm{a}}$ University of Manchester, UK ${ }^{\mathrm{b}}$ University of Surrey, Guildford, UK ${ }^{\mathrm{c}}$ Eden Rehabilitation Centre, Cooroy, Queensland, Australia

First published on: 17 May 2010

To cite this Article Wilkinson, Ray, Bryan, Karen, Lock, Sarah and Sage, Karen(2010) 'Implementing and evaluating aphasia therapy targeted at couples' conversations: A single case study', Aphasiology, 24: 6, 869 - 886, First published on: 17 May 2010 (iFirst)

To link to this Article: DOI: $10.1080 / 02687030903501958$

URL: http://dx.doi.org/10.1080/02687030903501958

PLEASE SCROLL DOWN FOR ARTICLE

Full terms and conditions of use: http://www.informaworld.com/terms-and-conditions-of-access.pdf

This article may be used for research, teaching and private study purposes. Any substantial or systematic reproduction, re-distribution, re-selling, loan or sub-licensing, systematic supply or distribution in any form to anyone is expressly forbidden.

The publisher does not give any warranty express or implied or make any representation that the contents will be complete or accurate or up to date. The accuracy of any instructions, formulae and drug doses should be independently verified with primary sources. The publisher shall not be liable for any loss, actions, claims, proceedings, demand or costs or damages whatsoever or howsoever caused arising directly or indirectly in connection with or arising out of the use of this material. 


\title{
Implementing and evaluating aphasia therapy targeted at couples' conversations: A single case study
}

\author{
Ray Wilkinson \\ University of Manchester, $U K$ \\ Karen Bryan \\ University of Surrey, Guildford, UK \\ Sarah Lock \\ Eden Rehabilitation Centre, Cooroy, Queensland, Australia \\ Karen Sage \\ University of Manchester, $U K$
}

\begin{abstract}
Background: In recent years conversation has become an area of interest for aphasia therapy, with several studies using conversation analysis (CA) to target and evaluate therapy. Most of these studies have focused on the main conversation partner of the person with aphasia, and in particular have targeted the partner's pedagogic behaviours in relation to the person with aphasia. Evaluations of therapy have primarily taken the form of qualitative analyses of change in conversational behaviours.

Aims: This single-case intervention study aims to advance research into interaction-focused intervention for aphasia in the following ways: by targeting intervention at the person with aphasia and the main conversation partner as a couple; by focusing on conversational behaviours where the person with aphasia can be seen to be restricted by the conversational actions of the conversation partner, in particular by recurrent questioning using closed questions and yes/no interrogatives; and by using a novel combination of qualitative and quantitative approaches to evaluate the intervention.

Methods \& Procedures: CA was used to target and evaluate interaction-focused intervention for a couple where one partner has aphasia. Evidence for change was evaluated using qualitative and quantitative evidence of change in conversational behaviours; evidence from naïve raters of pre- and post-intervention conversation extracts; and interview/other feedback from the conversation partner.

Outcomes \& Results: There was evidence that the intervention had changed the couple's conversational behaviours. In particular, the conversational behaviours of the non-aphasic partner were in general less restricting for the person with aphasia in that she was now using fewer questions and more instance of other types of turns, such as paraphrases. Following intervention the person with aphasia had also changed in that he was now producing turns that had more sentences, or attempts at sentences, and which developed the topic of talk across several of his turns.
\end{abstract}

Address correspondence to: Ray Wilkinson, Neuroscience and Aphasia Research Unit (NARU), School of Psychological Sciences, University of Manchester, Zochonis Building, Oxford Road, Manchester M13 9PL, UK. E-mail: ray.wilkinson@manchester.ac.uk

This research was funded by the Tavistock Trust for Aphasia.

(C) 2010 Psychology Press, an imprint of the Taylor \& Francis Group, an Informa business http://www.psypress.com/aphasiology

DOI: $10.1080 / 02687030903501958$ 
Conclusions: The study provides evidence that directly targeting the conversational behaviours of the person with aphasia and/or a main conversational partner can produce positive change, and can achieve this in a way that is ecologically valid. In particular, it highlights the usefulness of targeting conversational behaviours that are proving to be maladaptive for the participants. It provides further evidence that creating change in the non-aphasic partner's conversational behaviour may facilitate change in the person with aphasia's conversational and linguistic performance.

Keywords: Aphasia; Therapy; Conversation; Couples.

The ultimate aim of therapy for people with spoken language impairments due to aphasia is to improve their ability to communicate and interact with significant others in daily life. One way in which this issue has begun to be addressed is by focusing aphasia therapy on conversation (Hopper, Holland, \& Rewega, 2002; Kagan, Black, Duchan, Simmons-Mackie, \& Square, 2001; Simmons-Mackie, Kearns, \& Potechin, 2005). There have been a small but growing number of aphasia therapy studies that have drawn on the method and findings of conversation analysis (CA) (Hutchby \& Wooffitt, 2008) in order to implement therapy targeted at the everyday conversational behaviours of the person with aphasia and a significant other (usually a spouse) and to evaluate that therapy (see Wilkinson, submitted, for an overview of these interactionfocused intervention studies). In this paper we present the findings of a single-case therapy study that used CA as the basis for targeting and evaluating intervention.

This study adds to the existing literature in a number of ways. First, the person who has been the target for therapy in these interaction-focused therapy studies has typically been the non-aphasic conversation partner (Booth \& Perkins, 1999; Lesser \& Algar, 1995; Simmons-Mackie et al., 2005; Turner \& Whitworth, 2006). In this study therapy was targeted at, and carried out with, the person with aphasia and the wife of the person with aphasia as a couple. While some other conversation-focused therapy studies have targeted both partners as a couple (e.g., Boles, 1997; Cunningham \& Ward, 2003), these studies involved differences from the one presented here. The conversations recorded in the Cunningham and Ward (2003) study, for example, were less "naturally occurring" than those used in our study. Topics were suggested to the participants before their conversations and the participants were encouraged to use props such as magazines, catalogues and an atlas that had been left for their use. In the Boles (1997) study the therapy provided for four couples where one partner had aphasia was based primarily on linguistic analyses of word frequency, utterances in T-units, and repair-type behaviours of each participant. The analysis in the study presented here focuses on how the couple collaborate on a turn-by-turn basis to produce interactional sequences of talk together, with a particular focus on question-answer sequences.

Second, a main theme of previous studies has been to work with the non-aphasic partner to cut down or stop using certain pedagogic behaviours in conversation that have led to displays of negative emotions such as upset, frustration, or embarrassment in the couple's conversations (for exceptions, see Lesser \& Algar, 1995, and Sorin-Peters, 2004). These pedagogic behaviours have included test questions (i.e., the non-aphasic partner asking questions to which he/she already knows the answer) (Burch, Wilkinson \& Lock, 2002), and "correct production sequences" (Lock, Wilkinson, \& Bryan, 2001), i.e., stretches of talk where the non-aphasic partner encourages the person with aphasia to produce the correct version of a word, even when it is clear that both partners know what the target is (Booth \& Perkins, 1999; 
Booth \& Swabey, 1999; Lock et al., 2001; Turner \& Whitworth, 2006; Wilkinson et al., 1998). In this study we report intervention targeted at a different issue, i.e., where the couple post-onset of aphasia have developed a restricted style of talking that masks the competence of the person with aphasia and does not allow him (or in other circumstances, her) opportunities to make significant use of his remaining linguistic resources.

Third, since CA is primarily a qualitative approach that does not straightforwardly incorporate quantitative analysis (Schegloff, 1993), some therapy studies using CA have used a qualitative approach as the method of evaluating the intervention (Burch et al., 2002; Turner \& Whitworth, 2006; Wilkinson et al., 1998). Here we use a novel combination of qualitative and quantitative approaches to evaluate the intervention, which provides more robust evidence of positive change than a qualitative approach alone.

\section{METHOD}

\section{Participants}

The couple who took part in this study will be referred to as "Len" and "Jane". At the time of the study Len was 66 and had had a left cerebro-vascular accident (CVA) 18 months previously. He presented with a Broca-type aphasia and a right hemiplegia, and was able to walk with care and write with his non-preferred (left) hand. Jane, his wife, was 63 . Both were retired teachers.

\section{Conversation data: assessment and intervention}

Jane recorded the couple's conversation at home on two separate occasions prior to the intervention (termed here "Conversation 1" and "Conversation 2") and once after it ("Conversation 3"). The length of the two sets of conversation recordings prior to the intervention was 15 minutes 10 seconds and 9 minutes 28 seconds respectively, giving 24 minutes 38 seconds in total. The length of the conversation recorded after the therapy was 37 minutes 36 seconds. In each case the recording was made up of more than one conversation. The conversational data were analysed using CA methods and findings as outlined in Lock et al. (2001) with a particular focus on features of turn taking, turn construction, topic and repair (see Hutchby \& Wooffitt, 2008, for a description of these phenomena). Excerpts of around 8-10 minutes were transcribed in order to allow a more detailed analysis of certain sections (for CA transcription symbols see Hutchby \& Wooffitt, 2008; in the transcripts presented here, words in round brackets mark where those words are misproduced due to sound production problems and/or are unclear to the transcriber).

There were eight intervention sessions, which took place once a week at the couple's home and lasted between 1 and 2 hours. The intervention focused on changing the conversational behaviours of both Len and Jane and included:

- Jane to use open questions more often; to repeat or paraphrase what Len had just said; and to use minimal turns in the form of continuers (Hutchby \& Woofitt, 2008) such as "mm hm".

- Len to use the possibilities afforded by Jane's changes in conversational behaviour to add something new to the topic and to take an active role in conversation (for example, correcting Jane). 
TABLE 1

Raw scores on subtests of the Comprehensive Aphasia Test (CAT)

(Swinburn et al., 2004, pre-publication version)

\begin{tabular}{llrr}
\hline & & $\begin{array}{c}\text { Pre-intervention } \\
\text { scores }\end{array}$ & $\begin{array}{c}\text { Post-intervention } \\
\text { scores }\end{array}$ \\
\hline Cognition & Ravens coloured progressive matrices & $8 / 12$ & $8 / 12$ \\
& Pyramids and palm trees & $6 / 10$ & $8 / 10$ \\
& Verbal fluency & 4 & 5 \\
& Visual recognition & $10 / 10$ & $10 / 10$ \\
& Ideomotor apraxia & $7 / 12$ & $6 / 12$ \\
& Picture description & $5 / 10$ & $6 / 10$ \\
& Arithmetic & $5 / 6$ & $4 / 6$ \\
Language & $24 / 30$ & $22 / 30$ \\
& Auditory comprehension: single words & $26 / 30$ & $24 / 30$ \\
& Written comprehension: single words & $11 / 16$ & $13 / 16$ \\
& Auditory comprehension: sentences & $10 / 16$ & $15 / 16$ \\
& Written comprehension: sentences & $8 / 32$ & $6 / 32$ \\
& Repetition: single words & $9 / 48$ & $10 / 48$ \\
& Naming: noun pictures & $7 / 48$ & $4 / 48$ \\
\hline
\end{tabular}

\section{Results of other clinical assessments used}

Len was assessed on a range of clinical aphasia assessments. The results of the relevant assessments are presented below.

Comprehensive Aphasia Test (CAT): Cognitive and linguistic sections. The CAT (Swinburn, Porter, \& Howard, 2004: used here in a pre-publication version) was administered twice; at the same time as Conversation 1 (pre-intervention) and at the same time as Conversation 3 (post-intervention). The cognitive and linguistic sections of the CAT showed no change post-intervention. Table 1 shows the pre- and postintervention scores on the subtests of the CAT split between cognitive and linguistic functions, and clearly reflects a lack of change in these skills.

This is as expected, since at 18 months post-onset most significant spontaneous recovery of language is likely to have taken place and since no aspect of this intervention was targeted at changing Len's linguistic impairments. One finding from the CAT, which was significant for the interaction-focused intervention, was that on the spoken picture description task Len was able to produce sentences or attempt sentence structure. In the pre-intervention administration of the task Len described the picture in this way: "a man asleep and the /rai/ is (points) it . . . (ah) cup (points to book) $/ \mathrm{drk} / / \mathrm{kpts} / / \mathrm{frpt} /$ (cat) goin in the /blefə/ the gates are falling and recorder it on ... a plant". Len's output here has far more grammatical structure than is evident in his conversational talk with Jane.

Conversation Analysis Profile for People with Aphasia (CAPPA). The CAPPA (Whitworth, Perkins, \& Lesser, 1997) interview was carried out with Len and Jane together on the three occasions when conversations were collected from the couple. The CAPPA allows the interview responses to be quantified and compared pre- and post-intervention. Figure 1 shows the comparison of how the couple viewed changes in Len's conversation before and after intervention compared to his pre-morbid conversation. It suggests that, after the intervention, the couple's impressions were 


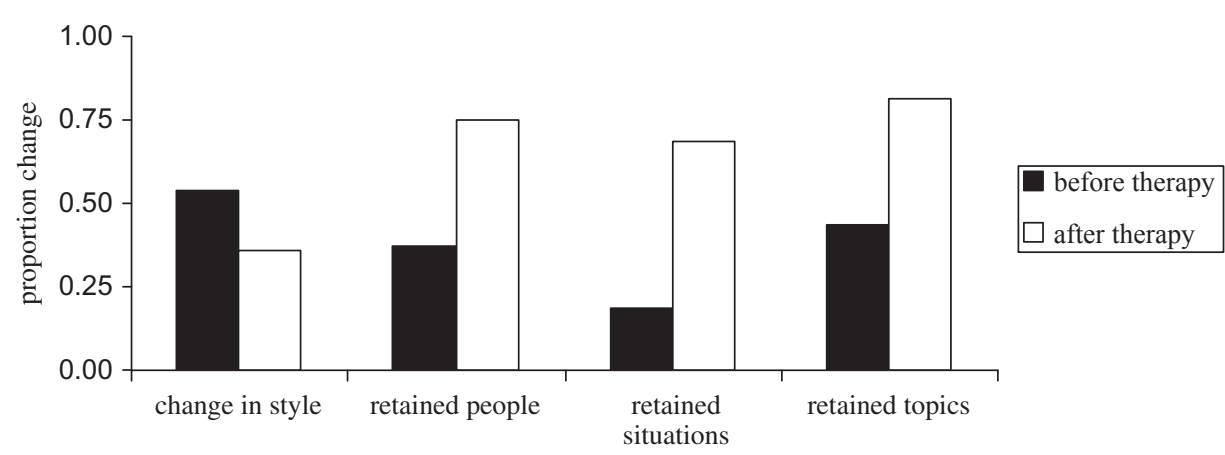

Figure 1. Conversation analysis profile for people with aphasia: Changes compared to pre-morbid conversation.

that Len was able to speak with more people in a wider range of places and on a wider range of topics.

\section{Quantitative comparisons of pre- and post-intervention conversations}

Conversation analysis (CA) was used in this study to compare the sequential patterns of talk in the couple's conversations pre- and post- intervention. As well as this qualitative analysis of change we also evaluated change post-intervention by carrying out quantitative comparisons of the pre- and post-intervention conversations.

Using pre- and post-intervention conversational extracts for quantitative comparison. Since the pre- and post-intervention conversational data were of different length, for the quantitative analysis we compared two continuous conversational extracts, one from Conversation 2 (pre-intervention data) and one from Conversation 3 (postintervention data). To select the best extract from the pre-intervention conversation data (Conversations 1 and 2), all of those data (i.e., 24 minutes 38 seconds in total) were divided up into eight segments of roughly equal length (these were roughly equal length because some segments coincided with natural breaks, such as the beginning or end of a conversation). Three speech and language therapists independently rated the eight video-recorded segments in terms of which one showed the best examples of the conversational behaviours that were later to be the targets for therapy. All three independently chose the same segment. In order to compare this segment with a segment from the post-intervention conversation, excerpts of both were transcribed into written extracts of an equal number of turns (35 turns). These pre- and post-intervention extracts are presented below as Extracts 2 and 3 respectively.

One way in which these pre-and post-intervention conversation extracts were used was to carry out a quantitative comparison of the conversational behaviours that had previously been examined qualitatively across the two conversations. Another way in which they were used was that they were presented to 15 outside raters (clinical speech and language therapists) for them to rate. The rationale and method for this part of the study will now be discussed.

Ratings of the pre- and post-intervention conversation sections by naïve raters. We were interested in finding out whether the changes that had been identified by the 
researchers on this project, who were familiar with the participants, the conversational data, and the conversation-analytic investigation of those data, could also be identified by raters who were naïve to all of these phenomena. There were two primary motivators for this part of the study. The first was the possibility that we, as the research team who were so closely aligned to the data, might be unconsciously identifying and arguing for particular changes between the pre- and post-intervention conversation data that were not evident to others outside the research project. This is one possible risk of using qualitative data analysis when arguing for change as a result of the intervention. The second motivator was to examine the following question: If naive raters could see a change in the two conversation extracts at a gross level, would they also be able to identify the presence of specific behaviours that made up that overall appearance of difference between the two extracts?

Specifically, the questions we examined were:

1. Could 15 naive speech and language therapists select, from the two extracts of conversational data, which extract was taken from the pre-intervention conversation and which from the post-intervention conversation?

2. Was there a significant difference between the two extracts in terms of the conversational behaviours identified by the naïve raters?

Selection of speech and language therapists to carry out the two task analyses. A total of 15 local speech and language therapists were recruited to take part in the analysis. On average the group had been qualified for 11.20 years ( $S D$ 9.63), with a range from 1 year to 37 years. The average time spent working with people with aphasia varied from 1 year to 27 years with a mean of 9.63 years $(S D 9.53)$.

Method for two task analyses. The first task required of the therapists was to watch the two video extracts from which the two 35-turn conversation transcriptions had been made, and to decide which extract was before intervention and which after. Therapists were not told what sort of intervention was provided. Therapists watched both extracts twice and were asked to write down which one they believed was from the pre-intervention conversation and which from the post-intervention conversation. They were also invited to comment on why they had selected their choice if they wished to do so. Their choices were then removed and collected before the second task was carried out.

The therapists were then told what the intervention had consisted of and were given written transcripts of the two extracts on which to mark down, using a number or letter code, their observations of specific conversational behaviours. These were subdivided into three behaviours from Jane (use of open questions; use of repeats or paraphrases of what Len had just said; use of minimal turns such as "mm hm") and two behaviours from Len (adding something new to the topic; taking an active role in conversation in some other way, e.g., by correcting Jane). The therapists again watched each video extract twice, first to note down Jane's behaviours and then on the second viewing to note Len's behaviours.

\section{Interview and other feedback from Jane}

Following the intervention Jane was interviewed about her experiences of taking part in the project. Post-intervention, Jane also spontaneously wrote the research team a letter about Len's progress. Both of these forms of feedback provide insights into 
Jane's view of the couple's experience of the intervention programme and will be presented below.

\section{RESULTS}

\section{Conversation pre-intervention}

A strongly recurring pattern throughout the two pre-intervention conversations was question and answer sequences where Jane asked a question, Len produced or attempted to produce an answer, and Jane followed Len's answer with a further question. Extract 1 (from Conversation 1) is an example of the type of talk made up of questioning sequences that recur throughout the two pre-intervention conversations.

Extract 1: Pre-intervention conversation (from Conversation 1).

01 Jane: did you have a good walk?

02 Len: (4.5) ((Len turns from Jane and looks forward; goes as if to speak then stops))

03 Jane: did you enjoy your walk?

04 Len: ${ }^{\circ}$ yes ${ }^{\circ}$

05 Jane: was it very hot?

06 Len: yes.

07 Jane: did you see any $\underline{b o d y ?}$

08 Len: (0.5) uh: no. yeah, (1.9) (man) with a dog.

09 Jane: you saw the man with the dog uhm $(.)^{\circ}$ whats his name. not Bill. can't remember what his name is ${ }^{\circ}$. right. was he going for a walk with the dog?

10 Len: no

11 Jane: oh $\lceil$ (right)

12 Len: L(he's) coming (down).

13 Jane: he was coming down, right.

14 Len: Lyeah

15 Jane: and uhm you didn't see Glen or Ellen?

16 Len: (0.2) y:es,

17 Jane: oh right. who did you see?

18 Len: (hh) (1.5) $\lceil$ (two) uh L ((holds up two fingers $)$ )

19 Jane: you saw them both.

20 Len: yeah

21 Jane: oh right. and what was- was Ellen going to the shops?

22 Len: no,

23 Jane: what was she doing?

The fact that so many of Jane's turns are questions can be seen to be constraining for Len in a number of ways. As a first pair part of an adjacency pair (Hutchby \& Wooffitt, 2008), questions are constraining in that they place the recipient of the question in the position of being expected to produce a certain type of action in the next turn, i.e., a second pair part in the form of an answer (Schegloff, 2007). If we examine the form of Jane's questions, however, it can be seen that Len is even more constrained. Many of the questions are in the form either of "wh"-type questions which demand a specific piece of information (as in turn 17: "who did you see?") or, in particular, are what Raymond (2003) terms "yes/no interrogatives" (turns 01, 03, 
$05,07,09,15,21,23)$. These are a type of question that constrains the linguistic form available to the answerer, in that the expected form of an answer would contain a "yes" or "no" (or an equivalent token such as "uh huh" or "nope" etc.) (Raymond, 2003). As such, utterances produced in response to these questions may typically display evidence of what Schegloff (1996) terms "positionally sensitive grammar", i.e., where the grammar of the utterance can be seen to be affected by its sequential position within the conversation, such as here in its role as an answer to a particular type of question.

Answerers can, of course, "escape" from these constraints of action and form to some extent. They may, for example, produce an answer to a yes/no interrogative that does not contain a "yes"- or "no"-type token (Raymond, 2003), or produce another action as well as, or instead of, the answer requested by the question. However, such responses are likely to entail more linguistic work (see Raymond, 2003), and this might be difficult for Len to achieve on a regular basis.

As such, the majority of Len's turns in these conversations are answers to Jane's questions, as is evident in Extract 1. In terms of the linguistic form of Len's utterances in the pre-intervention conversations, it is notable that he uses few sentences or attempted sentences in his talk. This is despite the fact that he is able to produce, or attempt to produce, sentences in other types of speech activity, such as the CAT picture description. In Extract 1, for instance, he produces one sentence ("(he's) coming (down)" in turn 12). Most typically, however, his utterances use a minimal form sufficient to produce an answer. Thus he regularly answers yes/no interrogatives with a "yes" or a "no" alone (turns 4, 6, 16, 20, 22) and "wh"-type questions with the minimal required information (turn 18). Where he does elaborate, it is typically where there is an expectation to do so (see Raymond, 2003), such as when his answer is different from that anticipated by Jane's question (turns 08 and 10/12), although even in this situation his response is often limited to "yes" or "no" (e.g., turn 16).

In response to Len's turns, it is notable that Jane in her next turn regularly produces another question. This may occur either by itself (turns 03, 05, 07, 23) or after some other kind of action earlier in her turn (turn 09, 17, 21). As such, there is a strong sequential pattern in these pre-intervention conversations of question-answerquestion or question-answer-response/question, which means that Len is almost constantly in the role of answerer and has very little opportunity to break out of that role in the talk. As a result of this, Len's role in terms of determining the content, direction, and topical development of these two conversations is a minimal one. Rather, his role in these and other aspects of these conversations is passive and responsive, with Jane constantly taking the initiating actions (such as asking questions and initiating and developing the topics of talk).

Extract 2 is the one from the two pre-intervention conversations that was judged by all three raters to be the "best", i.e., it is this section that the raters felt showed an overall better balance in the conversation, with Len producing more sentences and Jane's talk being less restrictive for Len:

\section{Extract 2: Pre-intervention conversation (from Conversation 2).}

01 Jane: so when I went (.) ehm (0.4) to the hospital with Nana this morning (0.8) what did you do

02 Len: I went eh (0.4) (wa ge gu) walk

03 Jane: you went for a walk (0.4) ehm (0.4) what time did you get up 
04 Len: (6.0) eh (se woo) (1.3) (wah) $(0.6)^{\circ} \mathrm{t}-\mathrm{t}-\left\lceil(\mathrm{tu})^{\circ}\right.$

05 Jane: Lbefore eight o'clock?

06 Len: (0.7) yes $(($ nods $))$

07 Jane: got up before eight o'clock (0.8) right and em (0.4) did you do your breakfast or did Andrew do it for $\lceil$ you

08 Len: ${ }^{\circ}$ ooh $^{\circ} \underline{I} \underline{\text { did }} \underline{\text { it }}$

09 Jane: you did it right did you make yourself a cup of tea=

10 Len: =ye:s $=(($ nods $))$

11 Jane: $=\operatorname{good}=$

12 Len: $=$ ooh (.) and I made (.) Andrew (.) (cup of) (.) tea

13 Jane: you $\mathrm{m}(\mathrm{h})$ ade Andrew a cup of $\mathrm{t}(\mathrm{h})$ ea heh heh (.) did you take it up

14 Len: no

15 Jane: heh heh heh ok(h)ay so he came down 「for his cup of tea

16 Len:

17 Jane: (0.5) so (.) what did you do then

18 Len: (1.4) went out for a walk

19 Jane: you went out for a walk (0.4) ehm (.) where did you go

20 Len: ooh (.) I-I (0.8) I: (0.6) went (.) to: (.) the: (0.7) ((points with finger, tuts and looks to Jane))

21 Jane: $\quad\left({ }^{\circ}\right.$ right $\left.^{\circ}\right)$ did you go up as far as Sou thfield?

22 Len:

23 Jane:

24 Len:

25 Jane:

26 Len:

you went to the end of Brig don'sLane?
Lye:s ((nods)) ye:s

${ }^{\circ} \mathrm{y}-\mathrm{ah} !{ }^{\circ}(($ points at Jane $)) \mathrm{y}-\mathrm{no}$

right okay you went to the end of Brigdon's $\lceil$ Lane

came back>

L yeah $(0.4)<($ and $)$ I

and you came back

27 Jane: and you

29 Jane: right straight back?

30 Len: ooh I: ((points forward)) k- (1.6) I: (.6) (wata) (0.6) ((unintelligible speech) $)($ points left $)$ ) (chaylim) (0.8) eh (cemetery)

31 Jane: you walked around the ccemetery

32 Len:

33 Jane:

34 Len

Lyes ((nods))

35 Jane: $\mathrm{mm}(0.4)$ did you go to look at grandad's grave?

Examples of Len producing, or attempting to produce, sentences are seen, for instance, in turns $02,08,12,18,20$ (incomplete due to the final location reference missing), 26 and 30 (although partly unintelligible and perhaps missing a verb). In part, this larger number of sentences and attempted sentences may be linked to Jane's production of more "open" (and hence less restrictive) questions in forms such as "what did you do" (turn 01) and "what did you do then" (turn 17). Len can also produce a sentence that is more than, or in addition to, the minimal form necessary at that point in the conversation, for example in his response to Jane's question in turn 30.

Significantly for the form of intervention that was later carried out with Len and Jane, one place where Len produces a sentence was in response to a non-question form by Jane, for example in turn 12 where he produces a sentence following Jane's assessment "good" in turn 11. Thus, on one of the relatively few occasions when Jane 
did not respond to an answer by Len with another question, Len was able to produce a turn that added to the topic and to what he had just previously said (in turn 10), and to do so in the form of a sentence. On two occasions, Jane also produced a paraphrase of what Len had just said rather than a new question (turns 27 and 31).

While Extract 2 shows some positive features compared to Extract 1, there are also many similarities between the two extracts. For example, Jane is still largely using her turns to produce questions (turns 01, 03, 05, 07, 09, 13, 15, 17, 19, 21, 23, $29,33,35)$. Len is still largely in the role of providing answers to questions. With the limited exceptions noted above, he is also contributing relatively little to the topical development and direction of the conversation.

\section{Intervention}

The intervention had three interlinked aims; two relevant for Jane and one relevant for Len. These aims emerged out of the investigation of the two pre-intervention conversations using $\mathrm{CA}$ and through discussion with the couple.

1. Jane would make greater use of forms to initiate sequences other than yes/no interrogatives and "closed" wh-type questions. The purpose of this change in Jane's conversational style was to make it less constricting for Len, and thus to provide him with more choices about the content and form of his turns (e.g., whether they should take the form of a sentence, or even more than one sentence).

2. Jane would respond to some of Len's turns with a repeat or paraphrase of his turn, or with a minimal turn such as "mm hm" (Hutchby \& Woofitt, 2008) rather than with another question. The purpose of this change was to provide Len with opportunities to add to his prior turn if he wished, and thus be more active in topic development in the conversation by building up more extended talk on a topic across his turns at talk.

3. Len would attempt to use the extra opportunities for talk that Jane's changes in conversational style would provide in order to contribute more to the conversation where relevant.

The intervention followed the three-stage therapy process described in the SPPARC (Lock et al., 2001). This involved:

1. working to raise the couple's/non-aphasic partner's awareness of relevant conversational behaviours in general (by, for example, using handouts, role plays, written exercises or video clips, and transcripts of other couples from the SPPARC);

2. working to raise their awareness of their own relevant patterns of conversational behaviours (by, for example, using video clips and transcripts from the couple's own conversations);

3. discussing and practising various strategies for changing relevant conversational behaviours (by, for example, using role plays in the therapy sessions or asking the non-aphasic partner to try a new behaviour between sessions and keep a written record of its success or otherwise for discussion at the next therapy session).

In the case of question behaviours, for example, Jane was introduced to findings about turns and sequences (such as questions and answers) using handouts adapted from those used in the SPPARC (Lock et al., 2001) that focused on different types of questions and the constraining effects on the recipient. 
To raise her awareness of her own patterns of questioning, Jane and the speech and language therapist observed selected video clips of Jane's questioning behaviours from the video recordings of the pre-intervention conversations. Handouts were also made for Jane, which included examples of her different types of questions and their effect on Len's responses. In discussion with the couple, Jane said that she felt her pattern of asking questions was helping Len to talk. She felt that she had "always been a bit of a teacher" and that this was behind some of her conversational behaviours observed on the video. In response the therapist suggested that, while Jane's style of asking many questions was indeed giving Len a space in the conversation to say something, he might be able to say more if he was given other types of opportunity to do so at certain points within conversation. The therapist discussed with Jane that she might want to take on less of a "teacher" role in conversation with Len. As a couple, they discussed an option whereby Jane would change her current style of talking to provide Len with opportunities to produce longer turns. It was stressed that if Len wanted to say more at these points in the conversation he then could do, but if he did not, Jane should not pressure him to speak. Len agreed with these points.

Strategies for change included paper and pen exercises where Jane had to analyse written versions of a range of questions she used in the pre-intervention conversations and decide which type of question each was (i.e., whether yes/no interrogative or another type of question). Jane was also encouraged to try different types of turns at home to initiate sequences (i.e., different types of questions, and statement forms instead of question forms) and also different types of turns in response to Len's turns (i.e., minimal turns or paraphrases of what Len had said rather than a further question). She was also encouraged to keep notes about what the effect of these different types of turn appeared to be on Len's talk. Jane and the therapist also engaged in role-plays together where the therapist took the part of Len, and Jane tried out different types of sequence-initiating and responsive turns in real time with the therapist. The therapist also videoed a role play between Jane and Len trying out their target conversational behaviours, and fed back to them about this. Each of these activities to facilitate change was preceded and followed by discussions between the therapist and the couple, in particular Jane, about the phenomenon being practised and how the participants felt about it.

Other parts of the intervention for the couple, which are not discussed here, included working to reduce the use of test questions by Jane and attempts to facilitate Len's writing skills within conversation.

\section{Conversation post-intervention}

In the post-intervention conversation there was evidence that the couple had changed their conversational behaviours in line with the aims of the intervention. Here we will provide four forms of evidence for these changes. These are: (1) qualitative changes in the sequential patterns in the pre- and post-intervention conversations; (2) quantitative comparisons of samples from the pre- and post-intervention conversations; (3) ratings by naïve speech and language therapists comparing samples from the pre- and post-intervention conversations; and (4) interview and other feedback from Jane on the couple's conversations post-intervention. These will now be presented in turn. 
Qualitative changes in the sequential patterns in the pre- and post-intervention conversations. There was evidence in the post-intervention conversation that the couple were now producing sequences of talk that differed from those seen anywhere in the pre-intervention conversations, and which were in line with the conversational behaviours discussed with the couple and practised by them during the intervention. An example of this can be seen in Extract 3:

Extract 3: Post-intervention conversation (from Conversation 3).

01 Jane: tell me about your walk.

02 Len: (2.0) I eh (1.9) I've eh (4.7) I (0.7) eh (walked) $\lceil$ (past the) (0.5) (cemetery)

(1.1) I (saw Glen)

L ((points outside) $)$

03 Jane: (1.1) you walked (.) past the cemetery (.) you s $\lceil$ aw Glen

04 Len:

(0.5) and he $\lceil$ (was) (0.7) (coming down)

L(saw Glen)

05 Jane: Glen was coming down.=

L((gestures towards himself $))$

06 Len: =yeah. (0.5) and he $\lceil$ have (bwocks) with him

L ((gestures carrying something $))$

07 Jane:

(0.9) a (with) eh- $(0.4)^{\circ}(\mathrm{him})^{\circ}(1.0) \Gamma^{\circ} \mathrm{eh}^{\circ}$

08 Len:

09 Jane: he had a bag with him right?, Lhe had a box $\lceil$ with him

10 Len: $\quad{ }^{\circ} \mathrm{ye}: \mathrm{s}^{\circ}(1.2)(($ gestures carrying something)) and he was (2.1) (going out) (0.5) t-to, (2.2) to eh, (2.6) ) oh (anyone oski) the (0.3) eh (0.5) (kowers but eh lays), ((laughs)) and k-heh heh and ((laughing)) $(0.5)$ he was (0.7) eh s: saying (0.6) eh (par) (0.5) eh (.7) ooh ((waves hand)) (0.9) (at) ((laughs)) eh (1.1) ooh ((makes gesture on lap-tray))

11 Jane: (1.5) eh he had a bag with him was he going shopping?

12 Len: no「((pointing))

13 Jane: Lcoming home from the $\lceil$ shops.

14 Len: $\quad$ Lno he was (0.9) he (wa) (ts)

(0.9) and he (wat) (.) talked to me about the (wales)?

15 Jane: he talked to Yyou- he talked to you about Wales (.) right?

16 Len: Lyeah

(0.3) and he (said), (0.7) but that wa- wasn't (3.1) (ay) eh (1.6)

17 Jane: and he said that,

18 Len: ah ah: ((growls and does dismissive gesture)) and he said eh (.) I (want aint a par) (0.8) $\lceil\underline{\text { ca:r. }}$

19 Jane: car. (0.4) right

20 Len: and he ((gestures pointing)) got (0.2) in the (.) (opposish).

21 Jane: he went in the car $\lceil$ (.) and he parked it opposite.

22 Len: Lno:

23 Jane: (0.7) no (0.4) did 「you say opposite (0.5) heh $\lceil$ heh

24 Len: $\quad$ ( (growls $)) \quad\lfloor$ (ay)

(0.8) ((moves hand left and right)) the (1.0) (cartin) (0.3) (poshtit)

25 Jane: (1.0) say that bit again=

26 Len: $=(\mathrm{e})(0.3)$ (wo) (.) (ps tk) ah (0.9) (2 syllables) (1.2) I (wo) (2 syllables)

(1.9) they (.) pa:k (.) it opposite. 
27 Jane:

28 Len:

(0.6) o-ppo-- site.

L yeah $(($ nods $))$

29 Jane: they parked the car oppo site?

30 Len:

31 Jane: right (.) somebody was in his space, was he annoyed?

L (enh) (.) yeah $(($ nods $))$

(0.5) 「heh heh (.) heh

32 Len: $\quad$ L( gestures 'don't know' $))^{\circ}$ no no ${ }^{\circ}$

33 Jane: no but he parked the car opposite.=

34 Len: $\quad={ }^{\circ}$ yeah $^{\circ}=$

35 Jane: =right (0.9) ehm had he been out in the car today then.

In turn 01 Jane starts the sequence not with a question but with a topic elicitor in the form of a statement ("tell me about your walk"). This is a non-restrictive form, which allows Len a range of choices about the content of his turn and what form it might take. It is also notable that following Len's turns Jane regularly responds with non-question forms, e.g., with a paraphrase or partial repeat of what she understands Len to have just said (turns 03, 05, 07, 21, 27), or with a repeat/paraphrase and a tag such as "right" (either with or without rising intonation) (turns 09, 15, 19). These turns by Jane are "other-oriented" (i.e., oriented to Len) in that they do not forward the conversation by Jane choosing to add some new topical content to the conversation (e.g., through a new question). Rather, these turns repeat or paraphrase something Len has just said and leave it to him to add something new to what he has previously said, and thus to direct the topical content of the conversation. Even though they are not paraphrases of what Len has just said, a similar phenomenon of other-orientation is seen in a number of Jane's other turns (i.e., the partial paraphrase/prompt in turn 17; the request for a repeat in turn 25; the re-statement of an already agreed element of the telling in turn 33).

The result of Jane's conversational behaviours is that Len has far greater opportunities to choose the content and form of his turns and to make use of the lexical and grammatical resources that he has available and which were evident, for example, in the CAT picture description. Len regularly takes these opportunities. For example, many of his turns contain sentences or attempts at sentences (turns 02, 04, 06, 10, 14, 16, 18, $20,24,26)$. In two cases his turns contain two or more sentences or attempts at sentences (turns 02 and 10), a phenomenon that never occurred in the two pre-intervention conversations. One way to consider this change in Len's grammatical production is in terms of Schegloff's (1996) notion of positionally sensitive grammar: while Jane's style of turn design in the pre-intervention conversations was restricting Len's ability to use the linguistic resources that he had available to him, the style she was now adopting post-intervention was less restrictive and allowed Len more opportunities to use these resources. These changes highlight the importance of considering language production, not simply in terms of an individual speaker, but rather in terms of that speaker within the context of other participants and their contributions to the conversation. It may be particularly important to analyse what type of utterance(s) occurred immediately prior to the speaker with aphasia's talk and to consider how the linguistic forms and social action evident in that talk might have been affected by the prior utterance(s).

Most notably, Len is now taking an active role in the conversation in ways that were not evident in the two pre-intervention conversations. First, he regularly actively develops the topic of the conversation in general and of his prior turn(s) in particular. This topical development carried out by Len can be seen across the following turns: Len informing Jane about where he walked and about meeting Glen 
(turn 02); that Glen was coming down and had a box with him (turns 04 and 06); other facts about Glen including what he said to Len (turns 10); this included talking about the Welsh rugby match (turn 14); something about his (Glen's) car (turn 18); and something about Glen parking his car opposite (turns 20, 24, and 26). This type of topical development by Len is quite unlike anything seen in his talk in the preintervention conversations and is directly linked to the changes in Jane's conversational behaviours. Len is also active in that he twice initiates a correction of Jane's understandings of what he has said (turns 08 and 22).

Quantitative comparisons of extracts from the pre- and post-intervention conversations. Based on the qualitative analyses provided above, Table 2 provides an overview of quantitative comparisons of the key conversational behaviours from the pre- and post-intervention extracts (i.e., Extracts 2 and 3). The comparison is made first in terms of how many of each speaker's turns contain an example of a key conversational behaviour. In both the pre- and post-intervention conversation samples Jane has 18 turns and Len has 17 turns.

Table 2 shows how the number of turns by Jane that contain questions to Len decreases from $78 \%$ to $22 \%$. Correspondingly, the number of her turns that do not contain questions to Len rises from $22 \%$ to $78 \%$. The differences in Len's sentence production, or attempted sentence production, are as follows. The number of his turns that contain at least one sentence, or attempted sentence, rises from $41 \%$ to $59 \%$, while the number of these turns that contain two or more sentences and/or attempted sentences rises from $0 \%$ to $17 \%$. (It is notable that it is not only this pre-intervention excerpt that contains no example of this phenomenon; rather, there are no turns by Len in the whole 24 minutes 38 seconds of the pre-intervention conversational data that contain two or more sentences or attempts at sentences). What this quantitative analysis does not capture, however, is the way in which Len's more grammatically complex utterances are now used post-intervention to develop the topic of talk in a manner that is quite different from anything observed in the pre-intervention data.

Ratings by naïve speech and language therapists comparing sections from the pre- and post-intervention conversations

Activity 1: Which section is pre-and which post-intervention? Of the 15 therapists, 14 correctly identified which section was from the pre-intervention conversation and which from the post-intervention conversation.

TABLE 2

Quantitative comparisons of extracts from the pre- and post-intervention conversations

\begin{tabular}{lcccc}
\hline $\begin{array}{l}\text { Key conversational behaviour in } \\
\text { extract of conversation }\end{array}$ & $\begin{array}{c}\text { Number } \\
\text { pre-intervention }\end{array}$ & $\begin{array}{c}\text { Number } \\
\text { post-intervention }\end{array}$ & $\begin{array}{c}\text { Percentage } \\
\text { pre-intervention }\end{array}$ & $\begin{array}{c}\text { Percentage Post } \\
\text { intervention }\end{array}$ \\
\hline $\begin{array}{l}\text { Jane: turns that contain } \\
\text { questions to Len }\end{array}$ & $14 / 18$ & $4 / 18$ & $78 \%$ & $22 \%$ \\
$\begin{array}{l}\text { Jane: turns that do not contain } \\
\text { questions to Len }\end{array}$ & $4 / 18$ & $14 / 18$ & $22 \%$ & $78 \%$ \\
$\begin{array}{l}\text { Len: turns containing at least one } \\
\text { sentence or attempted sentence }\end{array}$ & $7 / 17$ & $10 / 17$ & $41 \%$ & $59 \%$ \\
$\begin{array}{l}\text { Len: turns that contain two or } \\
\text { more sentences and/or } \\
\text { attempted sentences }\end{array}$ & $0 / 17$ & $2 / 17$ & $0 \%$ & $12 \%$ \\
\hline
\end{tabular}


TABLE 3

Mean $(S D)$ for all selected conversation behaviours pre- and post-intervention

\begin{tabular}{lccc}
\hline & Before therapy & & After therapy \\
& Mean $(S D)$ & & Mean $(S D)$ \\
\hline Total & $11.07(4.10)$ & \\
Jane & $6.93(2.60)$ & & $13.84(4.49)$ \\
Len & $4.13(2.85)$ & $10.33(3.33)$ \\
\hline
\end{tabular}

Total across the 35 turns and subtotals for Jane and Len.

Activity 2: Was there a significant difference between the two sections in terms of the conversational behaviours identified by the naïve raters? Table 3 shows the mean values and standard deviations for the number of times the therapists identified the five conversational behaviours in the pre- and post-intervention extracts (i.e., Jane using open questions, using repeats or paraphrases of what Len had just said, and using minimal turns such as "mm hm"; Len adding something new to the topic, and taking an active role in conversation in some other way, e.g., by correcting Jane). There was a significant change in the mean for all the behaviours (Wilcoxon matched pairs test: $\mathrm{W}=60$, two-tailed $p=.0007$ ) suggesting that the targeted therapy behaviours were observed in the conversation more in the post-intervention section than the pre-intervention section. The second part of the table indicates that this change was driven by both Jane's and Len's change in conversational behaviours as both showed significant differences in their pre- and post-intervention means (Jane: Wilcoxon matched pairs test: $\mathrm{W}=60$, two-tailed $p=.0007$; Len: Wilcoxon matched pairs test: $\mathrm{W}=52.50$, two-tailed $p=.0011$ ).

Post-intervention programme interview and other feedback from Jane on the couple's conversation post-intervention. In the post-intervention programme interview with Jane she commented on the changes she and Len had made in conversation due to the intervention they had received.

[On changes in her style of talking with Len] In my way of thinking about how do I talk to Len, I've changed in that way. As I've said, with the questions, with the statements, and he then had to think of something to say because, I wasn't going to go on and spoon feed him all the way along the line, which I think I did . . . And I think that's why when you said to me, "right, hang back a bit, let him do this", that he improved because of this. I mean he would start to talk and I would immediately jump in, and say so and so and so and so, you know, instead of letting him continue, slowly, in his own way.

[On changes in Len's language/conversation] He only spoke in word answers, I should say he had used sentences on about five occasions only in all that time that he'd had the stroke. But now he was beginning to use complete sentences.

A few months after the completion of the intervention programme, Jane also wrote an unsolicited letter to the research team to update them on Len's progress. Relevant sections are included below:

The main difference in Len's conversation since last June is that he now uses sentences daily instead of one word requests or answers. Len speaks to our son and my mother and his weekly telephone call to his brother is much less of a chore for Len. He attempts 
more conversation with friends. When Sarah first set up the recorder and stated that she wanted us to record our conversation my heart dropped. We had no conversation as I understood it. I would talk to Len constantly and apart from making sure that he understood me, I was content to leave it at that. When Len needed to tell me something we played "twenty questions" until I understood his needs. When we studied the tape we were both very surprised to see that Len was able to answer some questions. He gained in confidence through this experience and has gone from strength to strength. The initial sounds are sometimes wrong but Len is forming complete sentences, in his head, and attempts to talk in complete sentences. Len finds it easier to initiate a conversation relating to his needs ...

\section{CONCLUSION}

The focus of a significant number of previous research studies of interaction-focused intervention has been on changing pedagogic behaviours adopted by the conversation partner, such as test questions or correct production sequences (Booth \& Perkins, 1999; Booth \& Swabey, 1999; Burch et al., 2002; Lock et al., 2001; Turner \& Whitworth, 2006; Wilkinson et al., 1998). In this study we have discussed how an intervention programme was implemented and evaluated for a couple where the conversation partner had adopted a strongly recurrent pattern in conversation of using questions (in particular yes/no and closed questions) as a means of interacting with her husband with aphasia. This style of interaction was restrictive for the couple since, for example, it placed the person with aphasia in the position of recurrently providing answers, in particular short and linguistically limited answers such as "yes", "no" or a single word, and resulted in him adding little to the topical development of the talk. The fact that these conversational contributions by the person with aphasia recurrently displayed so few sentence structures or attempts at sentence production was notable since he was able to produce these in a picture description task.

The study provided evidence that interaction-focused intervention, which targeted particular conversational behaviours, particularly of the non-aphasic partner, was able to effect change in the conversational behaviours of both speakers. This included a larger production of sentences and attempted sentences by the person with aphasia. A result of this intervention was to allow the person with aphasia to use remaining linguistic resources in conversation, in particular grammatical resources, which had previously been suppressed by the conversational style adopted by the non-aphasic conversation partner (see also Simmons-Mackie et al., 2005).

The study used a novel combination of qualitative and quantitative approaches in order to evaluate the intervention. We suggest that this combination provides more robust evidence of positive change than would be provided by qualitative evidence of conversational change alone. There were four forms of evidence: both qualitative and quantitative evidence of change in the conversational behaviours produced by both the speaker with aphasia and the conversation partner; evidence from ratings by naive speech and language therapists comparing sections from the pre- and postintervention conversations; and evidence from the main conversation partner provided by an interview and an unsolicited letter.

The study highlights the importance for clinicians and aphasiologists of collecting everyday conversational data from the person with aphasia and a main conversation partner as part of an intervention programme. Without this form of data it is not possible to know which types of conversational behaviours one or both speakers may have adopted in everyday life in their attempts to adapt to conversations in the 
light of the person with aphasia's linguistic limitations. In some cases, one or more of the participants may adopt behaviours that appear to be maladaptive in that they, for example, highlight the person with aphasia's linguistic incompetence and lead to displays of negative emotion such as upset (Lock et al., 2001), or, as here, significantly restrict the person with aphasia's ability to use their remaining linguistic resources. This study suggests that when couples present with these maladaptive behaviours in conversation, these might be considered a priority for a therapy programme. One reason for this is that it may be easier and quicker to create noticeable change in participants' linguistic behaviour (including the person with aphasia's linguistic behaviour) by changing these maladaptive behaviours than by attempting to teach new linguistic or communicative behaviours. Since these maladaptive behaviours can often lead to negative emotions for participants, such as frustration or upset, dealing with these psychosocial issues first may also provide a more solid basis for the subsequent teaching and learning of new linguistic and/or communicative skills.

Finally, this study adds to a growing body of evidence showing that directly targeting the conversational behaviours of the person with aphasia and/or a main conversational partner can provide evidence of positive change. Notably, this change is ecologically valid in that it is evident not only within clinical testing or in interaction with a clinician or other professional, but rather can be seen in the participants' spontaneous conversational behaviour within their everyday lives.

\author{
Manuscript received 24 July 2009 \\ Manuscript accepted 16 November 2009 \\ First published online 17 May 2010
}

\title{
REFERENCES
}

Boles, L. (1997). Conversation analysis as a dependent measure in communication therapy with four individuals with aphasia. Asia Pacific Journal of Speech, Language and Hearing, 2, 43-61.

Booth, S., \& Perkins, L. (1999). The use of conversation analysis to guide individualised advice to carers and evaluate change in aphasia: A case study. Aphasiology, 13(4-5), 283-304.

Booth, S., \& Swabey, D. (1999) Group training in communication skills for carers of adults with aphasia. International Journal of Language and Communication Disorders, 34(3), 291-310.

Burch, K., Wilkinson, R., \& Lock, S. (2002). A single case study of conversation-focused therapy for a couple where one partner has aphasia. British Aphasiology Society Therapy Symposium Proceedings 2002 (pp. 1-12). London: British Aphasiology Society.

Cunningham, R., \& Ward, C. (2003). Evaluation of a training programme to facilitate conversation between people with aphasia and their partners. Aphasiology, 17(8), 687-707.

Hopper, T., Holland, A., \& Rewega, M. (2002). Conversational coaching: Treatment outcomes and future directions. Aphasiology, 16(7), 745-761.

Hutchby, I., \& Wooffitt, R. (2008). Conversation analysis: Principles, practices and applications (2nd ed.). Cambridge, UK: Polity Press.

Kagan, A., Black, S. E., Duchan, J. F., Simmons-Mackie, N. N., \& Square, P. (2001). Training volunteers as conversation partners using 'Supported Conversation for Adults with Aphasia' (SCA): A controlled trial. Journal of Speech, Language and Hearing Research, 44, 624-638.

Lesser, R., \& Algar, L. (1995) Towards combining the cognitive neuropsychological and the pragmatic in aphasia therapy. Neuropsychological Rehabilitation, 5, 67-96.

Lock, S., Wilkinson, R., \& Bryan, K. (2001). SPPARC (Supporting Partners of People with Aphasia in Relationships and Conversation): A resource pack. Bicester, UK: Speechmark Press.

Raymond, G. (2003). Grammar and social organization: Yes/no interrogatives and the structure of responding. American Sociological Review, 68, 939-967.

Schegloff, E.A. (1993). Reflections on quantification in the study of conversation. Research on Language and Social Interaction, 26(1), 99-128. 
Schegloff, E. A. (1996). Turn organization: One intersection of grammar and interaction. In E. Ochs, E.A. Schegloff, \& S. A. Thompson (Eds.), Interaction and grammar. Cambridge, UK: Cambridge University Press.

Schegloff, E. A. (2007) Sequence organization in interaction. Cambridge, UK: Cambridge University Press.

Simmons-Mackie, N. N., Kearns, K. P., \& Potechin, G. (2005). Intervention of aphasia through family member training. Aphasiology, 19, 583-593.

Sorin-Peters, R. (2004). The evaluation of a learner centred training programme for spouses of adults with chronic aphasia using qualitative case study methodology. Aphasiology, 18(10), 951-975.

Swinburn, K., Porter, G., \& Howard, D. (2004). Comprehensive Aphasia Test (CAT). Hove, UK: Psychology Press.

Turner, S., \& Whitworth, A. (2006). Conversational partner training programmes in aphasia: A review of key themes and participants' roles. Aphasiology, 20, 483-510.

Whitworth, A., Perkins, L., \& Lesser, R. (1997). Conversation Analysis Profile for People with Aphasia. London: Whurr.

Wilkinson, R. (2009). Conversation analysis and aphasia therapy. International Journal of Language and Communication Disorders. Manuscript submitted for publication.

Wilkinson, R., Bryan, K., Lock, S., Bayley, K., Maxim, J., Bruce, C., et al. (1998). Therapy using conversation analysis: Helping couples adapt to aphasia in conversation. International Journal of Language and Communication Disorders, 33, 144-149. 